\title{
EL JUEGO MOTOR Y LA PEDAGOGÍA DE LAS \\ CONDUCTAS MOTRICES \\ MOTOR GAMES AND PEDAGOGY OF MOTOR CONDUCTS
}

\author{
Dr. Pere Lavega Burgués \\ Instituto Nacional de Educação Física da Catalunha/INEFC
}

\section{Resumen}

La educación física interesada por hacer un uso pedagógico de las situaciones motrices encuentra en el juego un escenario privilegiado. Sin embargo, actualmente son muchos los docentes que actúan sin criterio ni rigurosidad en su actividad cotidiana cuando se trata de entender y aplicar el juego. En este artículo, se hace una revisión conceptual de los principales rasgos del juego (como práctica motriz lúdica y reglada), a la vez que se evidencian algunas de las principales preocupaciones de la Educación Física actual. Finalmente para vencer esta miopía disciplinar se propone usar las lentes de la praxiología motriz, interesada en desvelar las propiedades de los juegos y en ofrecer herramientas que se puedan aplicar en el contexto educativo diario. En este contexto se puede justificar que la estrella polar del uso educativo del juego se halla en la aplicación de una verdadera pedagogía de las conductas motrices.

Palabras-Clave: Praxiología motriz; Juego; Lógica interna; Conductas motrices.

\section{Resumo}

A Educação Física interessada por fazer um uso pedagógico das situações motoras encontra no jogo um cenário privilegiado. No entanto, atualmente são muitos os docentes que atum sem critério nem rigorosidade em sua atividade cotidiana quando se trata de entender e aplicar o jogo. Neste artigo, se faz uma revisão conceitual das principais características do jogo (como prática motora lúdica e regrada), ao mesmo tempo que se evidenciam algumas das principais preocupações da educação física atual. Finalmente, para vencer esta miopia disciplinar se propõe usar as lentes da praxiologia motriz, interessada em desvelar as propriedades dos jogos e em oferecer ferramentas que permitam aplicar no contexto educativo diário. Neste contexto se pode justificar que a estrela polar do uso educativo do jogo se encontra na aplicação de uma verdadeira pedagogia das condutas motoras.

Palavras-Chave: Praxiologia motriz; Jogo; Lógica interna; Condutas motoras.

\section{Juego y Educación Física: un escenario tan disperso como oscuro}


Acercarse a la comprensión conceptual del juego es un reto frustrado, si se pretende abordar en términos absolutos. El juego, en su condición de realidad compleja, se resiste ante cualquier intento de definición o de conceptualización categórica. Como si de una pastilla de jabón mojado se tratara, se muestra tan resbaladizo con aquellas posturas titubeantes, como con los planteamientos cerrados, arrogantes y categóricos.

Tal y como reconocía Cagigal (1959) se afirma que los conceptos que más capacidad metafórica poseen son los que primariamente representan una realidad más profunda, más rica, más elemental. Además el rastro que ha dejado el término juego en sus antecedentes etimológicos, ya sea en sus raíces latinas "iocus" "jocus" o en sus expresiones "ludus, ludere, lusus o ludicrum " (TRAPERO, 1971, MOLINER, 1982 o COROMINAS, 1984; LAVEGA 1996) confirman los múltiples significados y alusiones cotidianas que le ha caracterizado.

A esta condición polisémica que caracteriza al juego, se le tiene que añadir la dispersión que ha caracterizado el ámbito de la educación física, huérfana de abundantes contribuciones epistemológicas en torno a lo que debe ser su objeto de estudio y de aplicación. No es sorprendente encontrar autores que sin haber encontrado el "norte intelectual", después de beber en fuentes de consulta de distintas disciplinas o simplemente haciendo uso de su experiencia tan subjetiva como poco científica, utilicen sin criterio e indistintamente expresiones como

a) formas jugadas, juegos de reglas, juegos sin reglas (como si fueran lo mismo);

b) deportes que no son juegos, juegos menores, juegos deportivos, juegos predeportivos (como si se pudiese establecer una jerarquía entre las prácticas motrices); 
c) juegos alternativos, deportes alternativos ( $\sin$ saber exactamente que significa hablar de alternativa, en cualquier caso nunca emular el planteamiento del deporte de alta competición);

d) juegos autóctonos, juegos tradicionales, juegos populares, juegos vernáculos (sin saber que significa realmente cada uno de esos adjetivos);

e) juegos deportivos en solitario o deportes individuales (poniendo al mismo nivel el atletismo, el tenis o el judo, sin saber que en estos dos últimos templos es necesario interactuar con otra persona para jugar y por tanto nunca pueden ser prácticas individuales).

Sin embargo, lo más preocupante, es saber que esta miopía conceptual también se evidencia en el terreno de la intervención profesional, donde indistintamente del objetivo pedagógico que se plantee, se elige cualquier práctica o situación de juego sin criterio, sin orden ni justificación... estas actuaciones tan sólo sirven para acentuar esa visión torpe; que al fin y al cabo, están al mismo nivel que el intento de "querer apagar fuego con gasolina”.

En este escenario tan disperso como oscuro el reto está servido; urge encontrar al menos una estrella polar que permita vislumbrar el norte al hacer uso del juego en la educación física; es decir, que posibilite disponer de un ámbito epistemológico solvente, donde se pueda compaginar la teoría con la práctica, tal y como ya indicaba siglos atrás el escritor y filósofo Ramon Llull en su libro de Contemplación (1273?).

\section{La Utilidad o Inutilidad de querer Definir el Juego}

Como cualquier manifestación de condición social, tratar de definir en términos absolutos la esencia del juego es un desafío que no se puede asumir. Sin embargo, para hacer operativo el trabajo científico, en algún momento será necesario contar con una definición que sirva como punto de partida.

$\mathrm{Al}$ asumir el reto de mostrar la dispersión y la complejidad inherente a la naturaleza del juego, ya hace unos años (LAVEGA, 1996), se realizó una revisión exhaustiva de 
definiciones aportadas por estudiosos del juego (entre los que destacan, Agricol de Bianchetti, Bally, Bülher, Cagigal, Claparede, Dehoux, Fraiberg, Gualazzini, Hartmann, Huizinga, Klein, Loy, Margolin, Miranda, Navarro Adelantado, Ortega y Gasset, Olaso, Parlebas, Piaget, Roberts, Arth y Bursh, Russel, Schmitz, Slavson, Vygotsky, Winnicott, Wundt, Lin Yutang...) así como de aportaciones de distintas generaciones de alumnos de enseñanza primaria, secundaria y universitarios. El análisis de contenido ordenado y sistemático de una muestra exhaustiva de definiciones sobre el juego permitió confirmar los siguientes apartados:

\section{El juego es ante todo una manifestación activa}

La gran mayoría de autores destacan la condición dinámica del juego haciendo uso de distintos términos, aquí entendidos como sinónimos: realidad, fenómeno, ser, conducta, acción, movimiento, motricidad, situaciones motrices, práctica, ejercicio, esfuerzo, comportamiento, praxis, acto, impulso, actividad...

Se deduce una primera reflexión importante; si se quiere ser coherente en el uso del juego se tendrá que evitar jugadores espectadores, ya que el juego es ante todo actuar, sentir... fluir mediante la participación activa, es decir, a través de la acción motriz.

\section{La dimensión lúdica o el alma del juego}

Cuando se afirma que el juego es una práctica lúdica (o lúdicra), se está haciendo alusión a todo un conjunto de aspectos que difícilmente se pueden delimitar en coordenadas numéricas. Aunque en general, los autores deducen estos componentes desde distintas disciplinas, se puede afirmar que en definitiva se trata de términos de naturaleza cualitativa y fenomenológica, ya que en buena parte dirigen la atención al interior del protagonista que participa.

Cada partida o encuentro lúdico hace emerger en mayor o menor medida este tipo de aspectos. Después de realizar un exhaustivo análisis de contenido se han agrupado en diez apartados. En cada nivel se mencionan algunos ejemplos de los términos identificados que también deben entenderse como sinónimos.

- Voluntario, liberación, acción libre (Huizinga), voluntario, apetencia (G. Bally), valores de libertad ( Schmitz), movimiento libre (Roux). 
- Placentero, gratificación, placer moral (Chateau), alegría, diversión (Huizinga), satisfacción (Tilguer, Ruskin y Renzi), tiempo libre personal (Tilguer, Ruskin y Renzi), forma de felicidad (Ortega y Gasset), agradable $\left(^{* 1}\right)$, jolgorio $\left(^{*}\right)$, ameno(*).

- Espontáneo, indisciplinado, instintivo, impulsividad (Buytendijk), irracional (Huizinga), intuitivo (Marani), desafío a la racionalidad (Duvignaud), improvisación (*), fantasía (Klein).

- Desinteresado, intranscendente, afinal, placer funcional, gratuito, improductivo, sin fines exteriores, fin en sí mismo, infinitud interna (Scheuerl), lábil (Huizinga), superfluo (Huizinga), no serio (Huizinga), futilidad (Marani), inutilidad (Marani), sin metas extrínsecas (Garvey), intencionalidad cero (Duvignaud), intencionalidad vacía (Duvignaud), autotélico (Baldwin y Grosse), alegría funcional (M.Yela), invención gratuita (D'Agostino), objetivo no material $(*)$.

- Incierto, tensión (Cagigal), inseguridad, probabilidad, aventura (Haigis), formas cambiantes (Garvey), 'variopinto', cambiante (Ommo Gruppe), ser mutante (Duvignaud), maleable (Duvignaud), plasticidad (Duvignaud), fluctuante (Ciskszentmihalyi), metamorfosis (J.Miranda).

- Ambivalente (Duvignaud), oscilatorio, contraste (Huizinga), vaivén (J.Miranda).

- Consciente, decisión, compromiso, subjetividad impregnada de decisiones y sentido (Duvignaud), mundo autodirigido (K.L.Schmitz), autoergía (Roux).

- Orden (Huizinga), armonía, ritmo, estética (Schiller, Kant), , proporción (Cagigal), equilibrio (Cagigal), creativo.

- Ficticio, separado, como si (Huizinga), abstracción, fantasía, imaginación, orientación propia, absorción (Huizinga), representación simbólica (Frobenius), fines ficticios (Claparade), simulación (Spencer), fluir (Ciskszentmihalyi).

- Serio (Wallon), solemnidad (Huizinga), actividad necesaria (K.Groos), necesidad interior (Schiller).

\footnotetext{
${ }^{1}$ Los términos que se acompañan del símbolo $(*)$ son palabras aportadas por alumnos.
} 
En el ámbito de la educación física, cuando un autor carece de un posicionamiento disciplinar o epistemológico bien construido, a menudo se queda con la simple enumeración de algunos de estos aspectos cualitativos, que no sirven para aportar luz en la búsqueda de directrices a seguir en la tarea docente. Sin embargo, quizá el alma del juego, representada por este listado inacabado de apartados, puede contribuir a que el profesor de educación física reconozca la importancia de hacer emerger estos aspectos en cada situación de juego que se plantee. En caso contrario, si no se contemplan y por ejemplo, se coarta: la libertad para intervenir, la espontaneidad, la incertidumbre del resultado, la creatividad o lo serio que es cada instante para el protagonista, seguramente se estará menguando la dimensión lúdica de esa situación motriz, mermando su potencialidad educativa.

\section{Las reglas (o el cuerpo) del juego}

respetar (derechos y condiciones) por todos los que quieran intervenir. $\mathrm{La}$ naturaleza convencional del juego aporta una contribución pedagógica muy relevante, ya que se fundamenta en el respecto hacia los demás; en el aceptar voluntariamente el pacto de reglas pero al mismo tiempo estar obligado a seguir las prohibiciones y restricciones que imponen esas reglas.

Los estudiosos del juego suelen destacar la condición del juego como sistema de reglas empleando distintos términos: reglamento, creador de orden o convenio (Huizinga), sistema de reglas, estatuto, codificación o acuerdo ludomotor (Parlebas), código, normas (Marani), libertad hecha ley (Yela), límites internos (Schmitz), estatuto, ser convencional (Robles, Olaso).

A pesar de reconocer que el juego es un sistema de reglas, en la práctica muchos profesionales no siempre saben con certeza que significa esta expresión. Parlebas (1981) enseña muy sabiamente como las reglas establecen la frontera entre las prácticas que se pueden denominar casi-juegos (situaciones motrices sin reglas, abiertas, cambiantes en cada intervención) de las que son juegos (situaciones motrices 
codificadas que disponen de un estatuto) o incluso deportes (situaciones motrices regladas e institucionalizadas).

Al adoptar una visión sistémica y realizar un análisis de contenido de las principales aportaciones intelectuales referidas a las reglas, se deduce que éstas hacen referencia a los siguientes componentes del juego, entendidos como integrantes del sistema de reglas:

- Los jugadores (generalmente número y tipo de interacción motriz entre los protagonistas -colaboración, oposición, colaboración-oposición; roles a desempeñar...). También denominados: actores, protagonistas o personajes del juego.

- El espacio (dimensiones, características y modo de utilizarlo- zonas prohibidas, subespacios a franquear, distancias a respetar...). También denominado según diversos autores: acotación espacial, límite espacial (Huizinga), espacio cerrado (Huizinga).

- El material (características y forma de manipularlo). También indicado con los términos: soporte de juego, jugar con objetos (Klein, Isaacs, Lowenfeld), equipamiento (Fink), elemento mediador (Olaso) objetos extracorporales (Lagardera).

- El tiempo (inicio, secuencias temporales a seguir, finalización, unidades temporales a respetar...). Igualmente se mencionan otras expresiones sinónimas: acotación temporal, límite temporal (Huizinga), provisional (Huizinga), finalización frágil (Schmitz), imperativos temporales (Robles).

A diferencia de los elementos cualitativos, frágiles, efímeros y volátiles; las reglas establecen las condiciones a respetar sobre los componentes que al ser fácilmente identificables y permanentes en todos los juegos; constituyen la piedra angular desde la que construir los cimientos de la intervención pedagógica. Estudiar las relaciones, los procesos y los mecanismos de funcionamiento que activan los juegos es saber estudiar sus reglas.

Por lo que hemos justificado hasta el momento se puede identificar el juego que se utiliza en la educación física como una Práctica Motriz Lúdica y Reglada. 
El esfuerzo por elaborar una definición en torno al concepto de juego no tiene demasiado sentido, si no cumple la función de servir de lente que permita "alumbrar" el camino a seguir en su estudio y en la intervención sobre el terreno práctico.

La dispersión y la falta de fórmulas a seguir que evidencia el examen de las definiciones, también se observa en el estudio pormenorizado de las teorías del juego, apartado que por la extensión a respetar en este artículo no se va a tratar. ${ }^{2}$ En el fondo las distintas interpretaciones disciplinares sobre el juego formuladas desde la fisiología (Shaller, Lazarus, Schiller, Spencer...), la biología (Harvey Carr, Stanley Hall, Groos, Bally EiblEibesfeldt, Lorenz...), la psicología (Freud, Winnicott, Erikson, Claparede, Wallon, Chateau, Wund, Piaget, Vygotsky, Elkonin...), la sociología y la antropología (Malinowski, RadcliffeBrown, Firth, Gmelch, Langsley, Eifermann, Salter, Salamone, Raabe, Lance, Geertz, Turner, Huizinga, Caillois, Sutton-Smith,...) no dejan de ser contribuciones complementarias y periféricas para el profesor de educación física.

A pesar de aportar reflexiones interesantes y eruditas, no centran la atención en desvelar las constantes y el orden interno que caracterizan las situaciones de juego por las que la educación física se interesa. Una vez más, el escenario observado justifica la necesidad de buscar una estrella polar que nos indique el camino a seguir en medio de esa oscuridad conceptual. En este contexto va a ser necesario acudir al legado teórico y práctico (epistemológico) que ofrece la ciencia de la acción motriz creada por el profesor Parlebas.

\section{La Praxiología Motriz, Estrella polar para la Pedagogía del Juego}

Iniciábamos este artículo planteando la necesidad de conciliar la teoría con la práctica; de poco sirve presentar un simple repertorio de juegos sin saber que consecuencias originan sobre nuestros alumnos; igual que poca utilidad tiene la erudición intelectual que no se puede trasladar a las situaciones cotidianas de la escuela, el instituto o cualquier centro educativo.

La praxiología motriz al considerar como objeto de estudio las leyes, regularidades y mecanismos de funcionamiento que se desencadena en cualquier juego motor ofrece un

\footnotetext{
${ }^{2}$ En el caso de querer profundizar sobre este tema y observar que tipo de aplicaciones prácticas se pueden deducir de la revisión de las teorías del juego se sugiere consultar Lavega (1997).
} 
legado extraordinario para que todo maestro o profesor de educación física que conozca y quiera usar las "lentes" de esta disciplina sea coherente, riguroso y científico en su ejercicio profesional.

Permitidme, que como si se tratara de un juego que os invite a lanzar al aire algunas preguntas que a menudo se pasean por la cotidianidad del profesor de educación física:

- ¿Realmente disponemos de criterios para elegir que tipo de deportes introducir en la escuela y cuáles no? ¿A caso el fútbol, el baloncesto y el balonmano son más educativos que por ejemplo el béisbol, el críquet o el hockey? A lo mejor (peor) la respuesta depende excesivamente de la afición o nivel de cualificación del profesor sobre esas prácticas.

- ¿Qué lugar ocupa en nuestro proyecto pedagógico el juego respecto al deporte?¿ El juego debe aparecer antes o después que el deporte? ¿ Tiene sentido hablar de juegos predeportivos? ¿ y si habláramos de deportes pre-juegos? ¿Qué debe enseñarse antes el deporte de "masas", el deporte tradicional o el deporte alternativo? ¿Se puede establecer una jerarquía de situaciones motrices, en la que se distingan prácticas importantes de otras que son secundarias?. A lo mejor (peor) la respuesta nos remite una vez más, a la visión personal que tiene cada docente, fruto de sus gustos, de sus experiencias previas como jugador o deportista, de la moda del momento o de las influencias que recibe de los medios de comunicación. - ¿Produce los mismos efectos sobre el alumno, el voleibol, que el pañuelo, el atletismo, el bádminton o el juego del balón tiro? ¿Sabemos que estamos activando en la personalidad del alumno al elegir un tipo u otro de contenidos?

- ¿ Cuáles son los aspectos más interesantes a evaluar en las situaciones de juego, ya sean juegos o deportes? ¿realmente la condición física y el trabajo de la fuerza, la resistencia o la velocidad es lo más importante a considerar en la utilización del juego? En este sentido me pregunto si al hablar de contenidos tal y como sugiere la reforma educativa, se está en condiciones de diferenciar entre a) Condición física rendimiento y salud; b) Habilidad motriz; c) Juegos, actividades deportivas y deportes; y d) Expresión corporal. Realmente se confunden objetivos con contenidos; la condición física, el rendimiento y la salud son finalidades, propósitos y no contenidos, mientras que el resto de bloques son 
contenidos. Los criterios para distinguir los bloques no son de la misma naturaleza; ¿seguro que la habilidad motriz no se puede tratar en los juegos, los deportes o la expresión corporal? ¿Cuál es la frontera conceptual entre el juego y la expresión corporal; seguro que no tienen puntos en común...?; ¿qué son entonces los juegos dramáticos o expresivos?...

- ¿ Realmente los docentes inciden sobre la función educativa que tiene el pacto de las reglas?¿Es que la única vía de aplicar el juego consiste en sugerir a los alumnos un recetario más o menos numeroso de situaciones para que ellos simplemente se limiten a llevarlos a la práctica?...

Una vez más la dispersión, la oscuridad, la falta de criterio vuelven a aparecer en el horizonte del juego cuando se quiere utilizar en la educación física. Sin embargo, con el ánimo de ser optimistas, vayamos a apuntar algunos "puntos de luz" en el camino por el túnel en el que nos encontramos.

\section{El juego entendido como un sistema}

Pedro, Susana, Salvador y Toni son alumnos de $2^{\circ}$ de ESO que están jugando al balón prisionero (balón tiro, cementerio, brile, jugar a matar...). Cada uno de ellos se enfrenta a las mismas exigencias que establecen las reglas del juego. Las reglas de este juego establecen los límites de la práctica dentro de los cuales se genera un orden o lógica interna. Todos ellos al buscar la pelota van a poder: lanzarla, despejarla o esquivarla, pasarla. Estas respuestas son acciones motrices del juego del balón prisionero, entendidas como el resultado visible que emerge del conjunto de relaciones internas que estable este juego como sistema. Así el orden o lógica interna del cementerio exige a cualquier protagonista que se relacione de un modo singular con los otros jugadores (colaborando $\mathrm{u}$ oponiéndose), con el espacio (ocupando y utilizando determinados subespacios), con el material (manipulando la pelota de la forma permitida) y con el tiempo (respetando las secuencias temporales del juego, iniciando y finalizando la partida de un modo muy concreto).

A este conjunto de modos singulares de relaciones internas que demanda un juego y a sus consecuencias sobre la práctica se le denomina lógica interna. 
Cada juego dispone de una lógica interna específica que orienta a los protagonistas a resolver determinados problemas o adaptaciones. En un plano metafórico diríamos que todo juego dispone de una partitura (lógica interna), la cual muestra la composición y la distribución las notas musicales que al interpretarse harán emerger la música (acciones motrices). Cada nota tiene sentido entendida en la totalidad de la pieza musical; estamos ante un sistema en donde todo encaja y todo se relaciona. El músico experto al acudir al pentagrama sobre el que se describe la composición de una canción (juego), rápidamente descubre sus propiedades, su organización interna y las dificultades los intérpretes tendrán que resolver; (nos estamos refiriendo a su lógica interna).

\section{La Educación Física como pedagogía de las conductas motrices}

Vayamos de nuevo a nuestros protagonistas, algo cansados ya que llevan más de media hora jugando al balón prisionero. Pedro, Susana, Salvador y Toni a pesar de tener que adaptarse a la misma lógica interna que impone este juego se comportan de forma distinta. Susana calculadora siempre busca asegurar el pase a su compañero Pedro, quien a veces un tanto despistado es contactado por el balón enemigo de su rival Toni. Toni muy atrevido no para de asumir riesgos, esquivando una y otra vez balones que le lanzan sus adversarios. Salvador muy conservador siempre recorre los espacios más alejados tratando de no caer en ningún peligro.

El concepto de acción motriz (notas musicales) correspondiente al juego del cementerio se personaliza al introducir el término de conducta motriz. La manera en como Pedro, Susana, Salvador y Toni han interpretado la lógica interna del juego (pentagrama) se concreta con la forma específica de resolver cada situación de juego mediante acciones motrices personales, es decir conductas motrices ("forma de interpretar el instrumento musical y de cantar").

La Educación Física es una pedagogía activa, es decir, que educa a través de la práctica física, por lo que su implicación viene determinada por su intervención directa en el actuar del alumno, en sus conductas motrices asociadas a una implicación total de la persona, que actúa como un sistema inteligente, pues requiere la vivencia completa de la experiencia motriz que a su vez activa sus dimensiones cognitiva, afectiva y relacional. 
La manera subjetiva de realizar las acciones motrices de cada alumno se corresponde con el concepto sistémico y unitario de conducta motriz. Esto ostenta una importancia capital en la educación física ya que, sabedores de que la persona no aprende de modo parcial sino que capta estructuras globales, la noción de conducta motriz permite constatar el itinerario motor que sigue cada persona de modo diferenciado, pudiendo el pedagogo adaptarse a su singularidad.

Cada persona al jugar va dibujando a través de su biografía su propio itinerario motor, pero es el pedagogo, a través de sus observaciones, quien puede constatar esta evolución. De ahí que la observación, descripción y catalogación de conductas motrices tiene que constituir el arsenal didáctico y pedagógico de primer orden de todo profesor de educación física que desee actuar profesionalmente con congruencia. (LAGARDERA y LAVEGA, 2002).

\section{El juego portador de un sinfín de conductas motrices}

Cuando el profesor de educación física decide usar las "lentes" de la praxiología motriz, aparecen extraordinarias respuestas a sus preguntas cotidianas.

La clasificación de los juegos o situaciones motrices que aporta Parlebas (1981) tan desconocida como poco aplicada, establece unos ámbitos o dominios imprescindibles para la educación física. Los criterios de interacción motriz con los otros jugadores (compañero, adversario) y la relación con el medio (incertidumbre) atestiguan la riqueza motriz de las situaciones que originan los juegos.

Según el criterio de interacción motriz se distinguen dos grupos de juegos:

a) Los juegos psicomotores o en solitario cuya lógica interna no exige tener que interactuar con otras personas. Nadie puede molestarme ni ayudarme, únicamente dependo de mis virtudes. Estos juegos son muy interesantes para hacer emerger conductas motrices asociadas a la automatización de las respuestas, a la repetición y constancia, a la dosificación de las fuentes energéticas (cualidades físicas) y al control del cuerpo.

b) Los juegos sociomotores. Se distinguen tres subgrupos de situaciones:

- Juegos cooperativos cuya lógica interna orienta a los jugadores a respetar el pacto colectivo, a tomar decisiones de modo compartido, a automatizar acciones colectivamente y a estar en constante comunicación con los otros. 
- Juegos de oposición. Subdivididos a su vez en 1. según la estructura de interacción en duelos individuales (uno contra uno), uno contra todos y todos contra todos; 2.según las condiciones de oposición en duelos simétricos (mismo rol para los rivales) y duelos asimétricos (condiciones distintas); y 3 según la estabilidad en la relación con los demás, en oposición estable (no hay cambio de rivales) y oposición inestable (hay cambio de rival).

Las conductas motrices de estos juegos van asociadas a procesos de lectura e interpretación de las acciones de los demás, anticipación motriz, estrategia motriz, toma de decisiones, desafío o competición.

- Juegos de cooperación-oposición. Subdivididos a su vez en 1. según la estructura de interacción en: uno contra todos-todos contra uno; duelo de equipos, n-equipos; juegos paradójicos; 2. según la condición de la interacción: duelo de equipo simétrico y duelo de equipo asimétrico; y 3. Según la estabilidad en la relación con los demás, en juegos de cooperación-oposición estables (los jugadores no cambian de equipo o relación) y juegos inestables (los jugadores cambian de equipo o relación).

Según el segundo criterio de relación con el medio se distribuyen dos dominios o clases de juegos:

a) Juegos en un medio estable, en los que la relación del jugador con el espacio no genera incertidumbre, ya que el espacio es regular, conocido, dominado, estandarizado. Aquí las conductas motrices se asocian a la automatización, la repetición, el control y dominio del cuerpo. Es el caso de la mayoría de deportes.

b) Juegos en un medio inestable, en los que la relación del jugador con el espacio genera incertidumbre, ya que las condiciones del terreno cambian debido a su irregularidad, su fluctuación. Las conductas motrices se asocian a la toma de decisiones, la estrategia, la anticipación, la inteligencia motriz, la aventura.

Sirva esta síntesis de los principales dominios o ámbitos de las situaciones motrices que originan los juegos, para significar que existe un inagotable abanico de opciones de lógicas internas que pueden asegurar una excelente pedagogía de las conductas motrices. 
El "abc" de la praxiología motriz se halla en el conocimiento de la clasificación sistémica mostrada; tan sólo hace falta aplicarla con criterio para encontrar un tesoro increíble en el universo de situaciones que ofrece el juego.

¡Dejemos de lado si se juega con un balón, con un disco volador o con un bolo!; ¡huyamos de pretender justificar que alguno de estos dominios es más importante que los demás! y vayamos a hacer uso de todas las posibilidades que ofrecen los juegos, a favor de generar una optimización de las conductas motrices deseadas.

Como se ha afirmado cada dominio de los juegos es portador de un conjunto de procesos o exigencias asociado a su vez a grupo concreto de conductas motrices. Haciendo uso de estos principios el educador está en óptimas condiciones para ser coherente a la hora de seleccionar los juegos más apropiados para cada objetivo pedagógico que se proponga educar. Es entonces cuando el siguiente paso supondrá identificar en cada grupo de juegos las conductas motrices ajustadas (correspondientes a las exigencias de la lógica interna que se practica) y las desajustadas (desviadas de los procesos que hace activar la lógica interna de la práctica) para hacer un seguimiento individualizado de cada alumno. Mediante ese catalogo de conductas motrices, el profesor tan sólo tiene que ir registrando regularmente la tendencia de las conductas motrices que protagonizan sus alumnos para ejercer con congruencia una verdadera educación física, optimizando las conductas motrices ajustadas y modificando o sustituyendo las desajustadas.

En el ejemplo que hemos visto, sobre el juego del balón prisionero (juego de cooperación-oposición, duelo de equipo simétrico, estable y en un medio estandarizado) ante las intervenciones de: Susana muy calculadora, Pedro poco atento; Toni muy arriesgado y Salvador bastante conservador, las optimización de las conductas motrices requerirá intervenciones pedagógicas individualizadas de cara que todos ellos orienten sus respuestas hacia las conductas más inteligentes y eficaces en la lógica interna de ese juego. En definitiva el juego, el deporte al igual que Pedro, Susana, Salvador y Toni están a la espera de ese buen profesional de la educación física que al ponerse las lentes de la praxiología motriz, le de sentido a su intervención, orientando sus planteamientos hacia esa estrella polar que debe ser la pedagogía de las conductas motrices. Es entonces cuando en ese escenario se hará realidad "el placer de jugar, el placer de educar". 


\begin{abstract}
Phyisical Education interested in making pedagogic use of motor situation finds in games a privileged scenario. However, there are many teachers who work without criteria in their daily activity when they try to understand or apply games. In this article we offer a conceptual review of the main features of games as a regulated playful practice in motor skills. At the same time we point out some of our main worries in present day Physical Education. Finally, to overcome this myopia we propose the use of lenses for motor praxiology interested in unveiling the properties of the games and in offering tools that may be applied in the daily educational context. In this context we can justify that the polar star of the educational use of games is foun in the application of a true pedagogy of motor conducts.
\end{abstract}

Key Words: Motor praxiology; Motor game; Internal logic; Motor conducts motriz.

\title{
Referências Bibliográficas.
}

CAGigal, J. M. Aporías iniciales para un concepto del deporte. Citius, Altius, Fortius: estudios deportivos, v.1, n.1, p. 7-36, 1959.

LAVEGA, P. Del joc a l'esport. Estudi de les bitlles en el Pla d'Urgell (Lleida). Tesis doctoral Colección de tesis microfichadas núm 2915. Barcelona. Universidad de Barcelona, Barcelona,

LAVEGA, P. (1997) "The educational and recreational application of play and games from their respective levels of organisation" en 20th World Play Conference, ICCP y la Facultad de Motricidad Humana de Lisboa. Se puede consultar en www.praxiologiamotriz.inefc.es.

LAVEGA, P. (2000) Los juegos y deportes populares-tradicionales. Barcelona, Inde, 2000 .

LAVEGA, P.; LAGARDERA, F. Introducción a la Praxiología Motriz. Barcelona, Paidotribo, 2002.

PARLEBAS, P. Juego, deporte y sociedad. Léxico comentado de praxiología motriz. Barcelona: Paidotribo, 2002. 